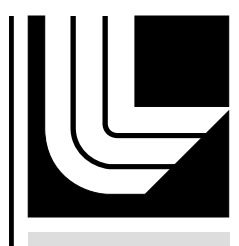

LA W REN CE LIVERMORE N A TIO NAL LABORATORY

Gyrokinetic Equations In An Extended Ordering

A. M. Dimits

November 20, 2009

Physics of Plasmas 
This document was prepared as an account of work sponsored by an agency of the United States government. Neither the United States government nor Lawrence Livermore National Security, LLC, nor any of their employees makes any warranty, expressed or implied, or assumes any legal liability or responsibility for the accuracy, completeness, or usefulness of any information, apparatus, product, or process disclosed, or represents that its use would not infringe privately owned rights. Reference herein to any specific commercial product, process, or service by trade name, trademark, manufacturer, or otherwise does not necessarily constitute or imply its endorsement, recommendation, or favoring by the United States government or Lawrence Livermore National Security, LLC. The views and opinions of authors expressed herein do not necessarily state or reflect those of the United States government or Lawrence Livermore National Security, LLC, and shall not be used for advertising or product endorsement purposes. 


\section{GYROKINETIC EQUATIONS IN AN EXTENDED ORDERING}

\section{Andris M. Dimits, LLNL}

We have developed a gyrokinetic theory in an extended ordering in which the small parameter is the ratio of the ExB shearing rate and the gyrofrequency. This allows for long wavelength ExB flows of order the thermal velocity instead of the more restrictive standard orderings which either require that the electrostatic or the ExB flow velocity be small compared with the thermal levels. Our theory generalizes prior work to allow for time dependence in the large long-wavelength component of the electric field, and a continuum of scales in the field components rather than just two distinct components. In the new theory, a significant part of the polarization drift now resides in the equations of motion, but there is still an identifiable polarization density that can be used to solve for the electrostatic potential from a quasineutrality or vorticity equation. 


\section{Introduction}

The presence of a strong ambient magnetic field in a plasma can, in many cases, be exploited to simplify the description of the plasma and the prediction of its behavior. In particular, if the gyro- (or "cyclotron" or "Larmor") orbital motion of a charged particle is much more rapid than the rate of change of the electromagnetic fields (and the rate of any "scattering" processes) seen by the particle, then the particle's magnetic moment becomes and adiabatic invariant. This fact was the basis for guiding-center theory [1] and, later, gyrokinetic theory [2-8]. In both cases, the temporal variation of the system is taken to be slow compared to the gyro frequency. In the guiding-center theory, the particle gyro-orbit size is required to be small compared to all spatial scales of any inhomogeneities in the system, while the gyrokinetic theory permits perturbations with scales comparable to the gyro orbit size.

Gyrokinetic-equation-based models have found wide use in the simulation of microturbulence and the resulting transport in magnetic fusion core plasmas [6, 9-17]. The gyrokinetic equations are valid under certain "gyrokinetic orderings," are faithful to the key kinetic (nonhydrodynamic) physics, but reduce the dimensionality of the relevant phase space by one relative to the raw "full-dynamics" Vlasov-Fokker-Planck equation. This reduction in dimensionality (e.g., from 6 to 5 for spatially 3-dimensional systems) can result in a large corresponding reduction in the number of degrees of freedom (grid cells, nodes, basis functions, or particles) needed to discretize the phase-space distribution (density) function to a given level of accuracy. Accompanying this reduction also is the removal of various high-frequency modes that are often not of central interest for the microturbulence or transport processes being simulated, but are numerically problematic 98$]$.

The success of gyrokinetic simulation of MFE core plasmas has motivated interest in extending the models to the edge and scrapeoff-layer regions $[18,19]$, as well as to other situations that stress the existing orderings. The length-scale separation between the radial plasma scales the gyroradius scales in the MFE edge region is much less than in the core. Thus, while we expect that a gyrokinetic ordering may still be satisfied in many edge and scrapeoff-layer situations, it will be less easily satisfied than in the core. Additional care therefore needs to be taken to ensure that the particular expression and use of the ordering results in a set of equations that are valid for the conditions and phenomena expected in the edge. Some progress in this direction has been made by several authors [20-24]. In [20], the derivation was extended to allow for electrostatic potential perturbations to be of order the temperature, and therefore relative perturbations of order 1 in the moments of the distribution function. However, this work did not allow for $E \times B$ flow velocities comparable to the thermal velocity. The work of refs. [21-24] allowed for such large $E \times B$ flow velocities, but only as a separate static long-wavelength component. We will show here that further extension of the ordering and applicability of the theory is possible.

The fundamental requirement for the magnetic moment to be an adiabatic invariant can be written as

$$
\varepsilon=\frac{\omega}{\Omega} \ll 1
$$


where $\omega$ is the rate of change of the electromagnetic fields seen by the particle and $\Omega=q B / M c$ is the cyclotron frequency. Here, $q$ and $M$ are the particle charge and mass, $B$ is the magnetic field strength, and $c$ is the speed of light. The magnetic moment can be written to lowest order as $\mu=M v_{\perp}^{2} / 2 B$, where is the perpendicular velocity.

Most of the nonlinear gyrokinetic theories [4-8] have as requirements that

$$
\frac{q \phi_{1}}{T} \sim \frac{\delta f}{F_{\text {eq }}} \sim k_{\|} \rho_{t} \sim \varepsilon \ll 1,
$$

Here, $\phi_{1}=\phi_{1}(\boldsymbol{x}, t)$ is the perturbed electrostatic potential, $T$ is the temperature, $\delta f$ and $F_{\text {eq }}$ are the perturbed and equilibrium distribution functions, and $k_{\|}$is the characteristic parallel wavenumber, $\rho_{t}=v_{t} / \Omega$ is the thermal gyroradius, $v_{t}=\sqrt{T / M}$ is the thermal (kinetic) particle speed, and $\varepsilon$ is the formal expansion parameter ("gyrokinetic smallness parameter.”) The first condition of Eq. (1.2) is somewhat counterintuitive, as it suggests that the theory would break down if a large constant were added to $\phi_{1}$. A partial resolution of this apparent paradox lies in that these theories also formally order $k_{\perp} \rho_{t} \sim 1$, where $k_{\perp}$ is the characteristic perpendicular wavenumber. Equation (1.2) also formally rules out the application of the resulting gyrokinetic system to situations where large perturbations are present, for example in the outer edge region of magnetically confined plasmas, even if the frequency ordering for adiabatic invariance of the magnetic moment is satisfied.

Some success has achieved in going beyond this ordering. Dimits et. al. [20] extended the canonical Hamiltonian gyrokinetic theory [5,8] to allow for $q \phi_{1} / T \sim 1$, with the new small parameter

$$
\varepsilon_{\mathrm{V}} \sim \frac{V_{E \times B}}{v_{t}} \simeq k_{\perp} \rho_{t} \frac{q \phi_{1}}{T} \ll 1
$$

Here $V_{E \times B}$ is a characteristic $E \times B$ drift velocity associated with $\phi_{1}$. Under this ordering, the electrostatic potential can have large long-wavelength components and small short-wavelength components, as well as components of intermediate sizes at intermediate scales. It has also been recognized [25] that this is also the ordering under which some of the iterative derivations of the gyrokinetic equations are valid.

Motivated originally by large core-plasma flows, the gyrokinetic formalism was extended to allow for a large long-wavelength field component $\Phi$ with, in addition to a short-wavelength component $\phi_{1}$ that satisfies the standard ordering [21-24]. The $E \times B$ drift associated with $\Phi$, $\left(\boldsymbol{u}_{E}=c \hat{\boldsymbol{b}} \times \nabla \Phi / B\right)$ can be of order $v_{t}$ provided that its scale length is macroscopic and its associated shearing rate is much less than $\Omega$. Such a two-component potential is not completely general, as it formally does not allow any mesoscale components. Also, the structure of the terms 
in the standard theories suggest that cross terms may be missing at second order in the results of Refs. [21-24], as will be shown in this work.

In the present paper, we derive a gyrokinetic theory based on the following more general ordering

$$
\frac{V_{E \times B}^{\prime}}{\Omega} \simeq\left(k_{\perp} \rho_{t}\right)^{2} \frac{q \phi_{1}}{T} \sim \varepsilon_{\mathrm{S}}=\frac{\omega_{\mathrm{L}}}{\Omega} \ll 1 .
$$

Here, $\omega_{\mathrm{L}}$ can be defined as the rate of change of the particle's perpendicular kinetic energy. Such an ordering makes intuitive sense in that if it is satisfied, then the perpendicular particle motion consists of gyromotion plus small perturbations to the gyromotion.

In order to carry out the derivation of the resulting gyrokinetic system, we use noncanonical Hamiltonian perturbation theory in a frame moving with a velocity field $\boldsymbol{u}(\boldsymbol{x}, v, t)$ that is chosen to make the perturbation theory work. In particular, by a suitable choice of $\boldsymbol{u}$, the gyrophase dependent part of the particle Lagrangian can be pushed out to orders no lower than $\varepsilon_{\mathrm{S}}^{1}$, and a perturbation theory can then be used to eliminate the remaining gyrophase dependences.

A clear exposition of the basic noncanonical Hamiltonian perturbation method, along with the application to the derivation of the drift-kinetic equations for a particle in an inhomogeneous magnetic field was given by R. Littlejohn [26]. This method was applied to the derivation of the gyrokinetic particle (characteristic), Vlasov, and Poisson equations for a plasma in a slab magnetic field by Hahm [7]. Subsequently, generalizations were made to a variety of situations, and this body of work has been reviewed by Brizard and Hahm [8]. The use of transformation to a (static but spatially dependent) moving frame was applied to the derivation of gyrokinetic equations by Brizard [21] and by Hahm [22]. In the present application, we will generalize this method to allow for the transforming velocity to depend on time as well as on the velocity itself. The temporal dependence brings additional terms into the equation of motion and facilitates the recognition of some of the terms in the results of Refs. [21-24] as parts of a polarization drift associated with $\boldsymbol{u}$.

We also note that even in our generalized ordering, it is still possible to iteratively solve the equations for the gauge functions, so that one is does not have to implement these equations directly in the form of additional kinetic equations of the type were given in Ref. [23].

Our theory is applicable in abitrarily boosted frames of reference, allows for time-dependent perturbations over a continuum of scales ranging from macroscopic to gyroradius, including large mean flows and large perturbations at macroscopic scales.

We anticipate that our equations may facilitate better connection to the Braginskii equations and MHD. This is a topic for further exploration. 


\section{Gyrokinetic Equations in the Generalized Ordering.}

In the present work, we consider a slab plasma in a uniform time independent magnetic field $\boldsymbol{B}=B \hat{\boldsymbol{b}}$, (e.g., with $\hat{\boldsymbol{b}}=\hat{\boldsymbol{z}}$ ) and electrostatic potential perturbations $\phi_{1}=\phi_{1}(\boldsymbol{x}, t)$ such that the $E \times B$ velocity $V_{E \times B}=c \hat{\boldsymbol{b}} \times \boldsymbol{\nabla} \phi_{1} / B$ satisfies $V_{E \times B}^{\prime} / \Omega \ll 1$, where $V_{E \times B}^{\prime}$ is the characteristic magnitude of the spatial derivatives of $\boldsymbol{V}_{E \times B}$. We will also require and use that $\frac{1}{\Omega}\left(\frac{\partial}{\partial t}+\boldsymbol{V}_{\text {ExB }} \cdot \boldsymbol{\nabla}\right) \ll 1$ when applied to $\boldsymbol{V}_{E \times B}$, even though $\frac{1}{\Omega} \frac{\partial}{\partial t}$ and $\frac{1}{\Omega} \boldsymbol{V}_{\mathrm{ExB}} \cdot \boldsymbol{\nabla}$ need not be small (e.g., when applied to gyroscale perturbations).

We will derive a gyrocenter Lagrangian (or Poincare'-Cartan 1-form) in a manner similar to that of Littlejohn [27]. In particular, a single pass through the perturbation expansion is used. In most gyrokinetic derivations [5,7,8,20-25], a guiding-center Lagrangian is first derived in the absence of the (gyroradius-scale part of the ) perturbed potential, and then perturbation theory is used to eliminate the gyrophase dependences that result from the perturbed potential. This is an important distinction because in our ordering, the electrostatic potential perturbations already appear at the lowest order in the theory.

We will use the method of noncanonical Hamiltonian perturbation theory in a locally moving reference frame [21]. However, our results can be greatly simplified if a slight generalization with respect to the method in Ref. [21] is used to allow the reference-frame velocity itself to depend on the velocity variable. Given the raw "physical" phase-space coordinates $Q=(\boldsymbol{x}, \boldsymbol{v})$, where $\boldsymbol{x}$ is the position and $\boldsymbol{v}$ is the velocity variable, the phase-space Lagrangian for a particle moving in an electromagnetic field with the magnetic vector potential $\boldsymbol{A}(\boldsymbol{x}, t)$ and electrostatic potential $\phi_{1}(\boldsymbol{x}, t)$, generalized to allow for transformation to a frame moving locally with a velocity $\boldsymbol{u}(\boldsymbol{x}, \boldsymbol{v}, t)$ is

$$
L(Q, \dot{Q}, t)=[\boldsymbol{A}(\boldsymbol{x}, t)+\boldsymbol{u}(\boldsymbol{x}, \boldsymbol{v}, t)+\boldsymbol{v}] \cdot \dot{\boldsymbol{x}}-\left\{\frac{1}{2}[\boldsymbol{u}(\boldsymbol{x}, \boldsymbol{v}, t)+\boldsymbol{v}]^{2}+\phi_{1}(\boldsymbol{x}, t)\right\} .
$$

The $v$ Euler-Lagrange equations

$$
0=\frac{d}{d t}\left(\frac{\partial L}{\partial \dot{\boldsymbol{v}}}\right)=\frac{\partial L}{\partial \boldsymbol{v}}
$$

give

$$
0=\left(\boldsymbol{I}+\boldsymbol{\nabla}_{\boldsymbol{v}} \boldsymbol{u}\right) \cdot[\dot{\boldsymbol{x}}-(\boldsymbol{u}+\boldsymbol{v})] .
$$

If $\left\|\boldsymbol{\nabla}_{v} \boldsymbol{u}\right\| \ll 1$, as will be the case for our choice of $\boldsymbol{u}(\boldsymbol{x}, \boldsymbol{v}, t)$, then it follows that

$$
\dot{\boldsymbol{x}}=\boldsymbol{u}(\boldsymbol{x}, \boldsymbol{v}, t)+\boldsymbol{v} .
$$


This is a key result that says that $\boldsymbol{v}$ is the velocity in the moving frame even if the velocity of that frame (with respect to the laboratory frame) itself depends on $\boldsymbol{v}$ and on time.

The $\boldsymbol{x}$ Euler-Lagrange equations

$$
\frac{d}{d t}\left(\frac{\partial L}{\partial \dot{x}}\right)=\frac{\partial L}{\partial \boldsymbol{x}}
$$

give

or, equivalently,

$$
\ddot{\boldsymbol{x}}=\dot{\boldsymbol{x}} \times \boldsymbol{B}-\nabla \phi_{1}-\frac{\partial A}{\partial t}, \quad \boldsymbol{B}=\boldsymbol{\nabla} \times \boldsymbol{A}
$$

$$
\dot{\boldsymbol{v}}=(\boldsymbol{u}+\boldsymbol{v}) \times \boldsymbol{B}^{*}-\nabla\left(\phi_{1}+\frac{1}{2} u^{2}+\boldsymbol{u} \cdot \boldsymbol{v}\right)-\frac{\partial \boldsymbol{A}^{*}}{\partial t}
$$

where

$$
\begin{aligned}
& \boldsymbol{B}^{*}=\boldsymbol{\nabla} \times \boldsymbol{A}^{*}, \\
& \boldsymbol{A}^{*}=\boldsymbol{A}+\boldsymbol{u} .
\end{aligned}
$$

The preceding results can be applied most effectively to the gyrocenter Lagrangian derivation as follows. Given $Q=(\boldsymbol{x}, \boldsymbol{v})$, and a choice of $\boldsymbol{u}(\boldsymbol{x}, \boldsymbol{v}, t)$ that will be specified shortly, a useful set of lowest-order guiding-center phase-space coordinates $Z=\left(\boldsymbol{R}, U_{\|}, \mu, \theta\right)$ can be defined as

$$
\begin{aligned}
& \boldsymbol{R}=\boldsymbol{x}-\boldsymbol{\rho}, \\
& U_{\|}=\hat{\boldsymbol{b}} \cdot \boldsymbol{v}, \\
& \boldsymbol{\rho}=\frac{1}{\Omega} \hat{\boldsymbol{b}} \times \boldsymbol{v}, \\
& \mu=\frac{v_{\perp}^{2}}{2 \Omega}, \\
& v_{\perp}=\left|\boldsymbol{v}_{\perp}\right|, \\
& \boldsymbol{v}_{\perp}=\hat{\boldsymbol{b}} \times \boldsymbol{v} \times \hat{\boldsymbol{b}},
\end{aligned}
$$

and $\theta$ is the angle between the vector $\boldsymbol{\rho}$ and some fixed direction perpendicular to the magnetic field. Now, define

$$
\langle\phi\rangle_{0}(\boldsymbol{R}, \mu)=\frac{1}{2 \pi} \oint d \theta \phi(\boldsymbol{R}+\boldsymbol{\rho})
$$

Then with the choice $\boldsymbol{u}=\frac{1}{\Omega} \hat{\boldsymbol{b}} \times \boldsymbol{\nabla}\langle\phi\rangle_{0}$, the transformation of Eq. (2.1) from phase-space coordinates $Q$ to $Z$, as given by Eqs.(2.2), gives 


$$
\begin{array}{rlr}
L= & \boldsymbol{A} \cdot \dot{\boldsymbol{R}}-\langle\phi\rangle_{0} & \ldots \ldots . O\left(\varepsilon^{-1}\right) \\
& +\left(\boldsymbol{u}+U_{\|} \hat{\boldsymbol{b}}_{0}\right) \cdot \dot{\boldsymbol{R}}-\mu \dot{\theta}-\left[\frac{1}{2}\left(U_{\|}^{2}+\boldsymbol{u}^{2}\right)+\mu \Omega\right] & \ldots \ldots . O\left(\varepsilon^{0}\right) \\
& +\boldsymbol{u} \cdot \dot{\boldsymbol{\rho}}-\delta_{1} \tilde{\phi} . & \ldots . . O\left(\varepsilon^{1}\right)
\end{array}
$$

Here,

$$
\delta_{1} \tilde{\phi}(\boldsymbol{R}, \boldsymbol{\rho})=\phi(\boldsymbol{R}+\boldsymbol{\rho})-\langle\phi\rangle_{0}(\boldsymbol{R}, \rho)-\boldsymbol{\rho} \cdot \nabla_{R}\langle\phi\rangle_{0}(\boldsymbol{R}, \rho)=O\left(\varepsilon=V_{E \times B}^{\prime} / \Omega\right),
$$

That $\delta_{1} \phi$ is of order $V_{E \times B}^{\prime} / \Omega$ can be seen from its Taylor series expansion

$$
\delta_{1} \tilde{\phi}(\boldsymbol{R}, \boldsymbol{\rho}) \approx \frac{1}{2} \rho^{2}\left(\hat{\boldsymbol{\rho}} \hat{\boldsymbol{\rho}}-\frac{1}{2} \boldsymbol{I}\right): \nabla_{R} \nabla_{R} \phi+O\left(\rho^{3} \boldsymbol{\nabla}_{R \perp}^{3} \phi\right)
$$

The terms in Eq.(2.3) are separated into terms formally of order $\varepsilon^{-1}, \varepsilon^{0}$, and $\varepsilon^{1}$. The only gyrophase dependent terms are at order $\varepsilon^{1}$. In assigning the orders of the terms, we used

where

$$
\boldsymbol{u} \cdot \dot{\boldsymbol{\rho}}=\frac{d}{d t}(\boldsymbol{u} \cdot \boldsymbol{\rho})-\dot{\boldsymbol{u}} \cdot \boldsymbol{\rho}
$$

$$
\dot{\boldsymbol{u}}=\left(\frac{\partial}{\partial t}+\dot{\boldsymbol{R}} \cdot \boldsymbol{\nabla}+\dot{\mu} \frac{\partial}{\partial \mu}\right) \boldsymbol{u}
$$

All of the derivative terms on the right hand side of Eq.(2.5) are of order $\varepsilon^{1}$, and the total time derivative term in Eq.(2.4) does not affect the equations of motion.

Also, Eq. (2.3) has $\mu$ dependences at first order through the dependences of $\langle\phi\rangle_{0}, \boldsymbol{u}$, and $\delta_{1} \phi$ on $\mu$. These dependences result in first-order corrections to $\dot{\theta}$.

A Lie-transform perturbative treatment is then applied to transform the phase-space coordinates $Z \rightarrow \bar{Z}=\left(\bar{R}, \bar{U}_{\|}, \bar{\mu}, \bar{\theta}\right)$, to eliminate the gyrophase dependence in the Lagrangian of Eq. (2.3) using $\delta_{1} \tilde{\phi} \sim \varepsilon \ll 1$ and $\left(\partial / \partial t+\boldsymbol{V}_{\text {ExB }} \cdot \nabla\right) / \Omega \sim \varepsilon \ll 1$. This latter property will used to iteratively solve for the gauge function $S_{1}$. Operators involving spatial derivatives have different orders depending what they operate on [20].

The primary result is the Lagrangian that gives the equations of motion. 


$$
\begin{aligned}
& L(Z, \dot{Z}, t)=\left[\boldsymbol{A}+U_{\|} \hat{\boldsymbol{b}}+\boldsymbol{u}+\frac{1}{2 \Omega} \frac{\partial}{\partial \mu}\left\langle\delta_{1} \tilde{\phi}(\nabla \boldsymbol{u}) \cdot \boldsymbol{\rho}\right\rangle\right] \cdot \dot{\boldsymbol{R}}+\frac{1}{2 \Omega^{2}}\left\langle\nabla \delta_{1} \tilde{\Phi} \times \hat{\boldsymbol{b}} \cdot \nabla \delta_{1} \tilde{\phi}\right\rangle \dot{\mu} \\
& -\left[\mu+\frac{1}{2 \Omega^{2}}\langle\nabla \tilde{\phi} \times \hat{\boldsymbol{b}} \cdot \nabla(\boldsymbol{\rho} \cdot \boldsymbol{u})\rangle \dot{\theta}-\left[\frac{1}{2} U_{\|}^{2}+\mu \Omega+\frac{1}{2} \boldsymbol{u}^{2}+\langle\phi\rangle\right.\right. \\
& \left.+\frac{1}{2 \Omega^{2}}\left\langle\nabla \tilde{\Phi} \times \hat{\boldsymbol{b}} \cdot \nabla \delta_{1} \tilde{\phi}\right\rangle-\frac{1}{2 \Omega} \frac{\partial}{\partial \mu}\left\langle\delta_{1} \tilde{\phi}\left(\delta_{1} \tilde{\phi}+\boldsymbol{\rho} \cdot \frac{\partial \boldsymbol{u}}{\partial t}\right)\right\rangle\right]
\end{aligned}
$$

Here, $\tilde{\phi}=\phi(\boldsymbol{R}+\boldsymbol{\rho})-\langle\phi\rangle(\boldsymbol{R}, \rho), \boldsymbol{u}=\frac{1}{\Omega} \hat{\boldsymbol{b}} \times \boldsymbol{\nabla}\langle\phi\rangle$, and the overbars have been dropped so that Z now represents the transformed phase-space coordinates. This Lagrangian has several new components at second order. This feature makes the equations of motion more complicated than in previous theories, but we show below that they are still tractable.

An observation that can be made immediately is that because $L$ is independent of $\theta$, the $\theta$ Euler-Lagrange equation yields the result that the quantity

$$
\mu_{\mathrm{AD}}=\mu+\frac{1}{2 \Omega^{2}}\langle\nabla \tilde{\phi} \times \hat{\boldsymbol{b}} \cdot \nabla(\boldsymbol{\rho} \cdot \boldsymbol{u})\rangle
$$

is conserved. $\mu_{\mathrm{AD}}$ is the magnetic-moment adiabatic invariant, up to the second order in our theory.

More insight into the transformed variables and the equations of motion can be gained through examination of the Lagrangian up to first order.

$$
L_{-1,0,1}=\left[\boldsymbol{A}+U_{\|} \hat{\boldsymbol{b}}+\boldsymbol{u}\right] \cdot \dot{\boldsymbol{R}}-\mu \dot{\theta}-\left[\frac{1}{2} U_{\|}^{2}+\mu \Omega+\frac{1}{2} \boldsymbol{u}^{2}+\langle\phi\rangle\right]
$$

Given a phase-space Lagrangian such as those of Eqs. (2.6) or (2.7), the equations of motion are obtained in the standard way. If we write formally

$$
L(Z, \dot{Z}, t)=\gamma_{i}(Z, t) \dot{Z}^{i}-H
$$

Then the Euler-Lagrange equations yield

$$
\omega_{j i} \dot{Z}^{i}=H_{, j}+\gamma_{j, t},
$$

where $\omega^{i j}=\frac{\partial \gamma_{j}}{\partial Z^{i}}-\frac{\partial \gamma_{i}}{\partial Z^{j}}$ are the components of the "Lagrange matrix" $\boldsymbol{\omega}$. Multiplying Eq.(2.8) by the "Poisson matrix" $\boldsymbol{P}=\omega^{-1}$ then gives

$$
\dot{Z}^{i}=P^{i j}\left(H_{, j}+\gamma_{j, t}\right) \text {. }
$$


Applying these results to the Lagrangian of Eq. (2.7) yields

$$
\begin{aligned}
& \dot{\boldsymbol{R}}=\boldsymbol{u}+\frac{1}{\Omega_{\|}^{*}} \hat{\boldsymbol{b}} \times\left(\frac{\partial}{\partial t}+\boldsymbol{u} \cdot \boldsymbol{\nabla}+U_{\|} \nabla_{\|}\right) \boldsymbol{u}, \\
& \dot{U}_{\|}=-\nabla_{\|}\langle\phi\rangle, \\
& \dot{\mu}=0, \\
& \dot{\theta}=-\Omega-\frac{\partial\langle\phi\rangle}{\partial \mu}+\frac{1}{\Omega_{\|}^{*}} \frac{\partial \boldsymbol{u}}{\partial \mu} \cdot \hat{\boldsymbol{b}} \times\left(\frac{\partial}{\partial t}+\boldsymbol{u} \cdot \nabla+U_{\|} \nabla_{\|}\right) \boldsymbol{u}, \\
& \boldsymbol{u} \equiv \frac{1}{\Omega} \hat{\boldsymbol{b}} \times \nabla\langle\phi\rangle, \quad \Omega_{\|}^{*}=\hat{\boldsymbol{b}} \cdot \nabla \times\left(\boldsymbol{A}_{g c}+\boldsymbol{u}\right)
\end{aligned}
$$

Thus, $\dot{\boldsymbol{R}}$ consists of a gyroaveraged $E \times B$ drift and a polarization drift. The effective magnetic field or gyrofrequency for the $E \times B$ drift is $\Omega$, while that for the polarization drift is $\Omega^{*}$, which is modified by the presence of vorticity in $\boldsymbol{u}$. This difference is a second order correction to those equations in (2.10) in which $\Omega^{*}$ appears. $\dot{\theta}$ has first and second-order modifications due to the $\mu$ dependences in $\langle\phi\rangle$ and $\boldsymbol{u}$. The terms

$$
\frac{1}{\Omega} \hat{\boldsymbol{b}} \times\left(\frac{\partial \boldsymbol{u}}{\partial t}, U_{\|} \nabla_{\|} \boldsymbol{u}\right)
$$

in $\dot{\boldsymbol{R}}$ are new and were not present in Refs. [21,22,24]. Note also, that the velocity field $\boldsymbol{u}$ in the present work contains both long- and short-wavelength components, and so is not the same as that in Refs. [21,22,24].

The equations of motion can be obtained to second order directly from the Lagrangian of Eq.(2.6), using Eqs.(2.8) and (2.9), and doing so takes advantage of desirable properties of the Hamiltonian formulation such as energy conservation. However, because of the many new noncanonical components that appear in the Lagrange matrix $\boldsymbol{\omega}$, the Poisson matrix $\boldsymbol{P}$ becomes quite dense and complicated. For the purpose of analysis and gaining insight, a calculation of the second-order equations of motion, through perturbative inversion of $\boldsymbol{\omega}$ is preferable. To do so, we use the fact that $\omega$ is easily separated into parts that have different orders in our expansion parameter

$$
\omega=\omega_{0}+\varepsilon \omega_{1}+\varepsilon^{2} \omega_{2}
$$

where $\omega_{0}$ is canonical, and both $\omega_{0}$ and $\omega_{0}+\varepsilon \omega_{1}$ are easily invertible. Using $\omega_{0}$ as the base for the perturbation expansion yields

$$
\boldsymbol{P}=\boldsymbol{P}_{0}+\varepsilon \boldsymbol{P}_{1}+\varepsilon^{2} \boldsymbol{P}_{2}+\ldots .
$$

with 


$$
\begin{aligned}
& \boldsymbol{P}_{0}=\omega_{0}^{-1}, \\
& \boldsymbol{P}_{1}=-\boldsymbol{P}_{0} \omega_{1} \boldsymbol{P}_{0}, \\
& \boldsymbol{P}_{2}=\boldsymbol{P}_{0}\left(\omega_{1} \boldsymbol{P}_{0} \omega_{1}-\omega_{2}\right) \boldsymbol{P}_{0} .
\end{aligned}
$$

Cleaning up Eqs.(2.10) to cleanly show terms of zero and first order, we obtain

$$
\left[\begin{array}{l}
\dot{\boldsymbol{R}}_{01 \perp} \\
\dot{z}_{01} \\
\dot{U}_{01 \|} \\
\dot{\mu}_{01} \\
\dot{\theta}_{01}
\end{array}\right]=\left[\begin{array}{c}
\boldsymbol{u}+\frac{1}{\Omega} \hat{\boldsymbol{b}} \times\left(\frac{\partial}{\partial t}+\boldsymbol{u} \cdot \boldsymbol{\nabla}+U_{\|} \nabla_{\|}\right) \boldsymbol{u} \\
0 \\
-\nabla_{\|}\langle\phi\rangle \\
0 \\
-\Omega-\frac{\partial\langle\phi\rangle}{\partial \mu}
\end{array}\right]
$$

and at second order,

$$
\left[\begin{array}{l}
\dot{\boldsymbol{R}}_{2 \perp} \\
\dot{z}_{2} \\
\dot{U}_{2 \|} \\
\dot{\mu}_{2} \\
\dot{\theta}_{2}
\end{array}\right]=\left[\begin{array}{c}
\frac{1}{\Omega} \hat{\boldsymbol{b}} \times\left\{\boldsymbol{\nabla}_{\perp} H_{2}-\left[\left(\boldsymbol{\nabla}_{\perp} \gamma_{2 \perp}\right) \cdot \boldsymbol{u}_{\perp}+\left(\boldsymbol{\nabla}_{\perp} \gamma_{2 z}\right) \cdot U_{\|}-\Omega \boldsymbol{\nabla}_{\perp} \gamma_{2 \theta}\right]-\left(\boldsymbol{\nabla}_{\perp} \times \boldsymbol{u}_{\perp}\right)_{z} \frac{1}{\Omega} \frac{d_{0} \boldsymbol{u}_{\perp}}{d t}\right\} \\
0 \\
-H_{2, z}+\left[\gamma_{2 \perp, z} \cdot \boldsymbol{u}_{\perp}+\gamma_{2 z, z} U_{\|}-\Omega \gamma_{2 \theta, z}\right]-\boldsymbol{u}_{\perp, z} \cdot \frac{1}{\Omega} \frac{d_{0} \boldsymbol{u}_{\perp}}{d t} \\
0 \\
-H_{2, \mu}+\left[\gamma_{2 \perp, \mu} \cdot \boldsymbol{u}_{\perp}+\gamma_{2 z, \mu} U_{\|}-\Omega \gamma_{2 \theta, \mu}\right]
\end{array}\right] .
$$

The Vlasov equation for the evolution of the gyrocenter distribution function, neglecting collisions, can be obtained in the standard way using the fact that the absence of dependence of Lagrangian on $\theta$ decouples the gyrophase dependent and independent parts of the Vlasov equation [5]

$$
\frac{\partial F_{i}}{\partial t}+\dot{\boldsymbol{R}} \cdot \nabla_{R} F_{i}+\dot{p}_{\|} \frac{\partial F_{i}}{\partial p_{\|}}=0
$$

The main ion contribution to the field equations is through the ion density $n_{i}$. This is obtained from $F_{i}$ via a "pullback transformation" [5] and integration over velocity space. The result, which relates $n_{i}$ to $F_{i}$, is 


$$
\begin{aligned}
n_{i}=\int \Omega_{\|}^{*} d Z \delta(\boldsymbol{R}+\boldsymbol{\rho}-\boldsymbol{x})\left[F_{i}(\boldsymbol{R}, \mu)+\delta_{1} \tilde{\phi} \frac{\partial F}{\partial \mu}\right] \\
\quad \approx \int \Omega d \mu d v_{\|}\left[F_{i}(\boldsymbol{x}-\boldsymbol{\rho}, \mu)+\nabla_{\perp}^{2} \bar{\phi} F_{i}+\delta_{1} \tilde{\phi} \frac{\partial F}{\partial \mu}\right]
\end{aligned}
$$

This differs from most previous results in some significant ways. The main polarization density is in second term on the last line of Eq.(2.12), which comes from the jacobian $\Omega_{\|}^{*}$ on the first line. This aspect is similar to what was noted in Ref. [22]. The third ("pullback”) term in Eq.(2.12), which in the standard gyrokinetic theories yields the main polarization density term, here gives only the finite Larmor radius (FLR) corrections to the polarization density.

\section{Summary and Discussion}

We have derived a set of gyrokinetic equations in an ordering that is a significant extension and generalization of those previously used. Although the derivation presented is for an electrostatic slab plasma, the results are already of interest because they contain several new terms and differences from previous theories. Furthermore the extension to include electromagnetic perturbations and general geometry should be straightforward because many of the key difficulties (e.g, the highly noncanonical nature of the terms in the symplectic part of the Lagrangian) are already present, and were addressed here.

Standard gyrokinetic theories, which were designed primarily with MFE core plasmas, use and required that $e \phi / T \ll 1$ [or in the electromagnetic case that $e \psi / T \ll 1$ where

$\psi=\phi_{1}-\left(p_{\|} / m c\right) A_{1 \|}$ ]. Prior work [20] involving one of the authors of the present paper gave a derivation of the theory that was valid under the ordering $e \psi / T \sim 1, \quad V_{E \times B} / v_{t h} \ll 1$, and this has also been recognized as a fundamental ordering for some of the iterative derivations (e.g., Ref. [25]). Motivated by the presence of large flow velocities in tokamak core transport barriers, an extension to allow for a large macroscopic scale-length component in $\Phi$ (which formally also had to be static), and which could have an associated $E \times B$ velocity $\boldsymbol{u}_{E}$ with $u_{E} / v_{t h} \sim 1$, was developed [21-24]. Our new ordering requires only that any rates of change of the perturbations, including the shearing rate of the $E \times B$ velocity are small. This ordering allows for perturbed fields that are significantly more general than those allowed by previous theories. These generalizations include:

(1) A continuum of spatial scales in the perturbations ranging from short (gyroradius-scale) perturbations all the way to the macroscopic scales.

(2) Time and parallel-coordinate dependence at all spatial scales, including the longest scales. In particular, it was possible to identify terms in the previous theories of Rerfs. [21-24] as parts of a polarization drift in the equations of motion. Our theory restores the 
partial time-derivative and parallel derivative contributions to this drift, which are of the same order as the $E \times B$ advective derivative terms that were kept in [21-24].

Our theory also reveals several new terms at the second order [e.g., in Eq.(2.6)] that result from the beating of such quantities as $\delta_{1} \tilde{\phi}$ and $(\nabla \boldsymbol{u}) \cdot \boldsymbol{\rho}$. We believe that the reason that these terms were absent from Refs. [21-24] was a result of the particular two-pass derivation process used in these theories, in which the guiding-center Lagrangian in the presence of the long-wavelength $E \times B$ flow was obtained first. This first step already involves gyroaveraging and a transformation that makes the Lagrangian independent of the gyrophase angle. When second Lie perturbation procedure was carried out in Refs. [21-24] to remove the gyrophase-angle dependence of the shorter scale perturbations, terms that result from the products of the gyrophase dependent parts of the long-wavelength and short-wavelength potentials were neglected, even though these are of the same order as the terms that were kept. These new cross terms are of the form

$$
\nabla \cdot\left\langle\boldsymbol{\rho} \cdot \nabla \boldsymbol{u}_{E} \delta \tilde{\phi}\right\rangle \sim \nabla\left\langle\left(\boldsymbol{\rho} \cdot \nabla u_{E}\right)^{2}\right\rangle \sim \nabla\left\langle\delta_{1} \tilde{\phi}^{2}\right\rangle
$$

and are formally of the same order as terms that are kept.

\section{Acknowledgments}

We wish to acknowledge useful discussions with J. Candy, B. Cohen, R. Cohen, G. Hammett, J. Krommes, T. Rognlien, R. Waltz and X. Q. Xu. This work was performed for U.S. DOE by LLNL under Contract DE-AC52-07NA27344.

References

[1] T.G. Northrop, “The Adiabatic Motion of Charged Particles,” Wiley Interscience Publishers, New York, 1963.

[2] P. H. Rutherford and E. A. Frieman, Phys. Fluids 11, 569 (1968).

[3] J. B. Taylor and R. J. Hastie, Plasma Phys 10, 479 (1968).

[4] E. A. Frieman and L. Chen, Phys. Fluids 25, 502 (1982).

[5] D. H. E. Dubin, J. A. Krommes, C. Oberman, and W. W. Lee, Phys. Fluids 26, 3524 (1983).

[6] W.W. Lee, Phys. Fluids 26, 556 (1983).

[7] T. S. Hahm, Phys. Fluids 31, 2670 (1988).

[8] A. J. Brizard and T. S. Hahm, Rev. Mod. Physics 79, 421 (2007).

[9] W. W. Lee, J. Comput. Phys. 72243 (1987).

[10] A. M. Dimits, T. J. Williams, J. A. Byers, and B. I. Cohen, Phys. Rev. Lett. 77, 71 (1996). 
[11] S. E. Parker, W. W. Lee, and R. A. Santoro, Phys. Rev. Lett. 71, 2042 (1993).

[12] W. Dorland, F. Jenko, M. Kotschenreuther, and B. N. Rogers, Phys. Rev. Lett. 85, 5579 (2000).

[13] A.M. Dimits, G. Bateman, M. A. Beer, B. I. Cohen, W. Dorland, G. W. Hammett, C. Kim, J. E. Kinsey, M. Kotschenreuther, A. H. Kritz, L. L. Lao, J. Mandrekas, W. M. Nevins, S. E. Parker, A. J. Redd, D. E. Shumaker, R. Sydora, and J. Weiland, Phys. Plasmas 7, 969 (2000).

[14]Z. Lin, T. S. Hahm, W. W. Lee, W. M. Tang, and R. B. White, Science 281, 1835 (1998).

[15] Y. Chen and S. E. Parker, Phys. Plasmas 8, 2095 (2001).

[16] J. Candy and R. E. Waltz, J. Comput. Phys. 186, 545-581 (2003).

[17] Y. Idomura, M. Wakatani, and S. Tokuda, Phys. Plasmas 7, 3551 (2000).

[18] X. Q. Xu, Phys. Rev. E 78, 016406 (2008).

[19] C.S. Chang, S. Ku, P.H. Diamond, Z. Lin, et al. Physics of Plasma, 16, 056108, (2009).

[20] A.M. Dimits, L.L. LoDestro, D.H.E. Dubin, Phys. Fluids B4, 274 (1992).

[21] A.J. Brizard, Phys. Plasmas 2, 459 (1995).

[22] T.S. Hahm, Phys Plasmas 3, 4658 (1996).

[23] H. Qin, R. H. Cohen, W. M. Nevins, and X. Q. Xu, Phys. Plasmas 14, 056110 (2007).

[24] T.S. Hahm L. Wang, J. Madsen, Phys. Plasmas 16, 022305 (2009)]

[25] F. I. Parra and P. J. Catto, Plasma Phys. Control. Fusion 50, 065014 (2009).

[26] R. Littlejohn, J. Plasma Phys 29, 111 (1983). 